\title{
Activités autour des 40 ans de présence de l'IFEA en Bolivie
}

Gérard Borras

\section{(2) OpenEdition \\ 1 Journals}

Édition électronique

URL : http://journals.openedition.org/bifea/7734

DOI : $10.4000 /$ bifea. 7734

ISSN : 2076-5827

Éditeur

Institut Français d'Études Andines

Édition imprimée

Date de publication : 1 décembre 2015

Pagination : 495-497

ISSN : 0303-7495

\section{Référence électronique}

Gérard Borras, "Activités autour des 40 ans de présence de l'IFEA en Bolivie », Bulletin de l'Institut français d'études andines [En ligne], 44 (3) | 2015, mis en ligne le 08 décembre 2015, consulté le 06 novembre 2020. URL : http://journals.openedition.org/bifea/7734 ; DOI : https://doi.org/10.4000/ bifea. 7734

\section{(c) $(1) \&$}

Les contenus du Bulletin de l'Institut français d'études andines sont mis à disposition selon les termes de la licence Creative Commons Attribution - Pas d'Utilisation Commerciale - Pas de Modification 4.0 International. 


\section{ACTIVITÉS AUTOUR DES 40 ANS DE PRÉSENCE DE L'IFEA EN BO LIVIE}

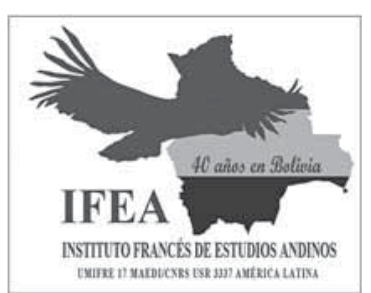

Cette année 2015 avait une valeur particulière pour notre activité scientifique dans la région andine. En 1975, Thierry Saignes, ethnohistorien, fut le premier pensionnaire de l'IFEA en Bolivie. Ce chercheur a profondément marqué la connaissance de l'histoire bolivienne et ses écrits sont une référence incontournable pour les études andinistes. II nous a semblé important de marquer cet anniversaire en faisant de 2015 l'année des 40 ans de l'IFEA en Bolivie. L'occasion de célébrer des décennies de collaborations avec nos partenaires dans ce pays, l'occasion aussi de rendre hommage à un chercheur ayant aussi marqué l'histoire de notre institution.

Nous avons mis en œuvre une programmation spécifique dont nous avons déjà en partie rendu compte dans les numéros précédents de ce Bulletin. Nous avons tout d'abord créé un logo particulier qui a été notre élément de communication institutionnel tout au long de cette année.

II nous semblait important que cette date anniversaire coïncide avec un hommage spécifique à Thierry Saignes. Grâce à Thérèse Bouysse-Cassagne et à notre partenaire éditeur Plural nous avons pu éditer un recueil de 18

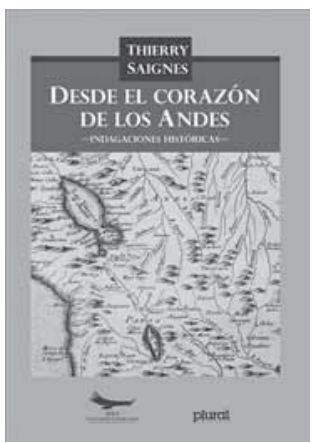
textesque nous considérionsimportants, parfois inédits en langue espagnole, souvent assez difficile d'accès. Cette nouvelle et importante collaboration Plural/IFEA a été présentée le 21 août dans les locaux de l'Alliance française de La Paz en présence de l'éditrice scientifique, Thérèse Bouysse-Cassagne, de Tristan Platt, du directeur de Plural, José Antonio Quiroga et de Gérard Borras, directeur de l'IFEA.

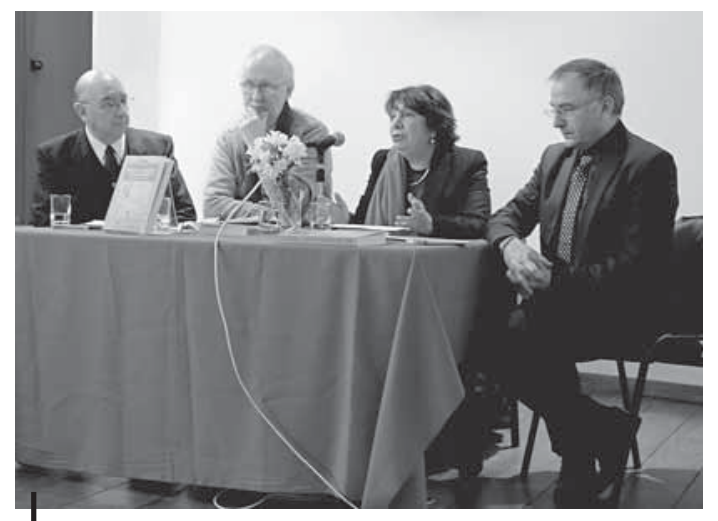

Présentation du livre de Thierry Saignes. De gauche à droite : José Antonio Quiroga, Tristan Platt, Thérèse Bouysse-Cassagne et Gérard Borras

Comme depuis plusieurs années, I'IFEA a été partenaire de la célèbre «Reunión Anual de Etnología» du M useo de Etnología y Folklore (MUSEF) de La Paz. Lors des deux dernières sessions de cette «Rébellion des objets » Elvira Espejo, la directrice du M usée, avait choisi la thématique des textiles, puis celle de la céramique. Pour cette troisième version, le Musef avait ciblé «el arte plumario ». Du 17 au 21 août des archéologues, des conservateurs, des fabricants, des utilisateurs, des juristes ont apporté leurs analyses, leurs témoignages. Car s'intéresser à cette pratique renvoit à une série considérable de questions : la conservation/restauration des pièces anciennes, leur mise en valeur muséographique, l'adaptation des pratiques contemporaines, le droit des populations à maintenir et conserver leurs traditions tout en respectant les diverses réglementations de protection de biodiversité et de 


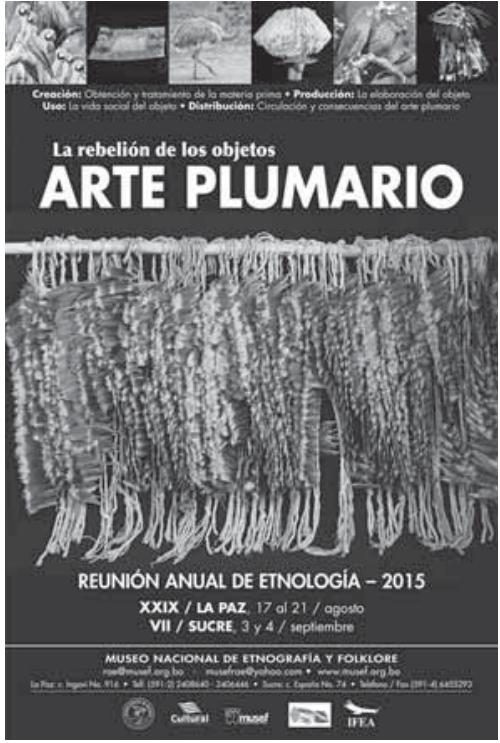

la faune sauvage. Pour l'occasion, le MUSEF avait mis en place une spectaculaire exposition et publié un catalogue d'excellente qualité qui vient s'ajouter aux deux précédents constituant ainsi un apport désormais incontournable sur l'importance de la culture matérielle dans le monde andin.

Dans le numéro du Bulletin précédent nous rendions compte du congrès «Arqueología y etnohistoria en losAndes y Tierras Bajas . Dilemas y miradas complementarias». Cet événement organisé par el Instituto de Investigaciones Antropológicas, le Museo Arqueológico de l'université Mayor de San Simón était appuyé par l'IFEA dans le cadre de sa programmation anniversaire. II s'est tenu à Cochabamba, dans les locaux du Centro Pedagógico y Cultural Simón I. Patiño, du 24 au 29 août. Lors de ces journées, une cinquantaine d'archéologues et d'historiens venant de onze pays différents ont présenté leurs travaux, débattu autour de quatre thématiques

- La méthodologie et l'interprétation en archéologie et

ethnohistoire

- L'interprétation et les « autres » sources

- Le pouvoir politique, les échanges et la territorialité

- Spiritualité, cosmovisions et rituels

Au cours de cet événément, les institutions et les collègues ont rendu un hommage mérité à trois grandes figures de l'histoire andine : Verónica Cereceda, Waldemar Espinoza et Teresa Gisbert.

La participation de I'IFEA au Congrès de l'Association d'Études Boliviennes du 21 au 24 juillet faisait aussi partie de cette programmation spécifique. Comme les années précédentes, nous avons contribué à ce congrès en proposant l'organisation d'un symposium consacré aux " objets musicaux, patrimoine, conservation, recherche » (cf. Bulletin 44/2 : 300-302). Plusieurs chercheurs ont partagé leur expérience dans ce domaine et envisagé une rencontre plus large qui aura lieu en 2016.

L'IFEA a signé en 2014 un accord de collaboration scientifique avec l'université Autonome de Barcelone. Ce programme co-financé a permis des avancées considérables dans le domaine de l'ethnohistoire. Pilar García Jordán et Ana Guiteras ont organisé cette année à Santa Cruz de la Sierra, les 14 et 15 octobre une table ronde dans le cadre du colloque sur Les Terres Basses (2èmes journées d'anthropologie, histoire et archéologie). Cet événement faisait aussi partie de la programmation anniversaire.

Plusieurs institutions étaient associées à cet événement : I'U niversité Autonome de Barcelone, le M usée d'histoire de l'U niversité Autonome Gabriel René Moreno de Santa Cruz, le programme post-grado d'histoire de I'U niversité Fédérale de Grande 


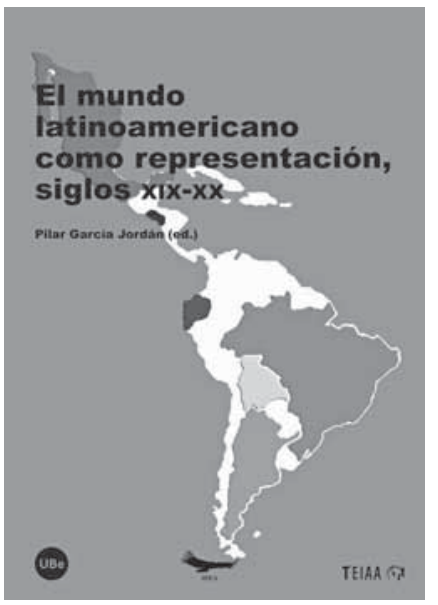

Dourados (MS, Brésil) et le Centre de Recherches « Frontière O uest : pouvoir, économie et Société » de l'université d'État du Matto Grosso.

Nous souhaitions aussi programmer une activité qui mettrait en avant les recherches des jeunes chercheurs de l'IFEA ou associés à I'IFEA travaillant en Bolivie, mais aussi donner un éclairage sur les travaux menés ces dernières années dans ce pays. La journée était structurée en trois volets. Tout d'abord la présentation orale des travaux et commentaires de collègues chercheurs. Marc-Antoine Vella, pensionnaire de I'IFEA, Vincent Nicolas, bénéficiaire de la bourse andine, Elise Gadea, Bourse d'aide à mobilité, Amélie Aubert Plard et Georgina Aurelia Chávez, bourse andine, ont présenté l'avancement de leurs recherches. Venait ensuite l'exposition de posters de recherches récentes. Philippe Erikson, Françoise Martinez, Alexis Pierrard, Andrea U rcullo, Marc-Antoine Vella, Céline G effroy, Francis Ferrié, N elson Ramos, Claudia Rivera Casanovas, se sont pliés à un exercice nouveau pour certains d'entre eux et qui a été largement plébiscité par le public.

Nous avons présenté enfin une rétrospective des collaborations éditoriales de I'IFEA en Bolivie en présence de José Antonio Q uiroga, directeur de Plural, notre principal partenaire éditeur dans ce pays.

Au final, l'IFEA aura participé et/ou organisé six événements scientifiques auxquels doivent s'ajouter les différentes conférences du réseau dans le cadre de l'U niversité de tous les savoirs. N ous y voyons le dynamisme d'une recherche qui aujourd'hui est en profond renouvellement. Les recherches en histoire, ethnohistoire, archéologie, anthropologie, sociologie, bénéficient du volontarisme deschercheurs confirmés et de la venue de jeunes professionnels qui assurent la relève. L'IFEA a, à sa manière mais de façon décidée, contribué à cette dynamique et continuera de le faire malgré la disparition prochaine de son poste de pensionnaire en Bolivie. C'est aussi le message que nous souhaitions faire passer en cette année 2015.

\section{Gérard BORRAS}

\section{CO LO Q UIO INTERNACIO NAL, ESTU DIANTES DE HISTO RIA DE LA PUCP 25 AÑOS CONSTRUYENDO HISTO RIA}

\section{Lima, 26-30 de octubre de 2015}

El Coloquio de Historia es, como escuché decir a la profesora Claudia Rosas, el más importante evento de la Especialidad de H istoria de la Pontificia U niversidad Católica del Perú. Es un momento del año donde tanto los alumnos como los profesores trabajan de la mano en, lo que se podría llamar, la presentación de la especialidad a la comunidad universitaria y a la crítica pública. Asimismo, es 